\title{
NEW GENERATING FUNCTIONS FOR CLASSICAL POLYNOMIALS ${ }^{1}$
}

\author{
J. W. BROWN
}

1. Introduction. As recently as 1951 Brafman [1]-[4] initiated a series of papers in which he gave a variety of new and unusual generating functions for ultraspherical polynomials. Our purpose here is to furnish two new classes of generating functions which include the ultraspherical case and thus add to Brafman's list. Our results are sufficiently general to yield analogous generating functions for the other classical polynomials containing parameters, those of Laguerre and Jacobi. All of the generating functions exhibited below appear to be new.

2. Preliminaries. Our discussion is in the language of formal power series and is based on the pair of binomial series transformations contained in the following lemma. This preliminary result is considerably more general than we need but is of interest in itself (see remarks in $\$ 4$ below).

Lemma. Given a sequence $\phi_{n}(n \geqq 0)$, define the new one

$$
\psi_{n}=\sum_{k=0}^{n}\left(\begin{array}{c}
\alpha+\beta n \\
n-k
\end{array}\right) \phi_{k} \quad(n \geqq 0) .
$$

Then

$$
\sum_{n=0}^{\infty} \psi_{n}\left[\frac{x}{(1+x)^{\beta}}\right]^{n}=\frac{(1+x)^{1+\alpha}}{1+(1-\beta) x} \sum_{n=0}^{\infty} \phi_{n} x^{n}
$$

and

$$
\sum_{n=0}^{\infty} \frac{\alpha}{\alpha+\beta n} \psi_{n}\left[\frac{x}{(1+x)^{\beta}}\right]^{n}=(1+x)^{\alpha} \sum_{n=0}^{\infty} \frac{\alpha}{\alpha+\beta n} \phi_{n} x^{n} .
$$

Proof. The proof is based on the well-known identities

$$
\sum_{n=0}^{\infty}\left(\begin{array}{c}
\alpha+\beta n \\
n
\end{array}\right)\left[\frac{x}{(1+x)^{\beta}}\right]^{n}=\frac{(1+x)^{1+\alpha}}{1+(1-\beta) x}
$$

and

Received by the editors June 8, 1968.

'In conjunction with NSF Grant GP-9140. 


$$
\sum_{n=0}^{\infty} \frac{\alpha}{\alpha+\beta n}\left(\begin{array}{c}
\alpha+\beta n \\
n
\end{array}\right)\left[\frac{x}{(1+x)^{\beta}}\right]^{n}=(1+x)^{\alpha}
$$

occurring as special cases of (2) and (3) respectively when $\phi_{0}=1$ and $\phi_{n}=0$ for $n \geqq 1$. Equations (4) and (5) appear, for example, in the classic of Pólya and Szegö [7, pp. 301-302] who obtained them by means of Lagrange's theorem. More recently, Gould [6] has rederived them by direct summation. To obtain (2), simply recall (1) and write

$$
\begin{aligned}
\sum_{n=0}^{\infty} \psi_{x}\left[\frac{x}{(1+x)^{\beta}}\right]^{n} & =\sum_{n=0}^{\infty} \sum_{k=0}^{n}\left(\begin{array}{c}
\alpha+\beta n \\
n-k
\end{array}\right) \phi_{k}\left[\frac{x}{(1+x)^{\beta}}\right]^{n} \\
& =\sum_{k=0}^{\infty}\left\{\sum_{n=0}^{\infty}\left(\begin{array}{c}
\alpha+\beta k+\beta n \\
n
\end{array}\right)\left[\frac{x}{(1+x)^{\beta}}\right]^{n}\right\} \phi_{k}\left[\frac{x}{(1+x)^{\beta}}\right]^{k} .
\end{aligned}
$$

Then, using (4) with $\alpha$ replaced by $\alpha+\beta k$, we arrive at the desired result. Equation (3) is similarly obtained by writing

$$
\begin{aligned}
\sum_{n=0}^{\infty} \frac{\alpha}{\alpha+\beta n} \psi_{n} & {\left[\frac{x}{(1+x)^{\beta}}\right]^{n} } \\
= & \sum_{n=0}^{\infty} \frac{\alpha}{\alpha+\beta n} \sum_{k=0}^{n}\left(\begin{array}{c}
\alpha+\beta n \\
n-k
\end{array}\right) \phi_{k}\left[\frac{x}{(1+x)^{\beta}}\right]^{n} \\
= & \sum_{k=0}^{\infty}\left\{\sum_{n=0}^{\infty} \frac{\alpha+\beta k}{\alpha+\beta k+\beta n}\left(\begin{array}{c}
\alpha+\beta k+\beta n \\
n
\end{array}\right)\left[\frac{x}{(1+x)^{\beta}}\right]^{n}\right\} \\
& \cdot \frac{\alpha}{\alpha+\beta k} \phi_{k}\left[\frac{x}{(1+x)^{\beta}}\right]^{k}
\end{aligned}
$$

and using (5) with $\alpha$ replaced by $\alpha+\beta k$.

3. Generating functions. Our new classes of generating functions follow readily from the above lemma.

THEOREM. The generalized hypergeometric polynomials

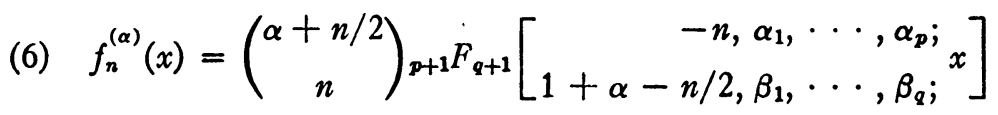

are generated by

$$
\sum_{n=0}^{\infty} f_{n}^{(\alpha)}(x) t^{n}=\frac{[1+u(t)]^{1+\alpha}}{1+u(t) / 2}{ }_{p} F_{q}\left[\begin{array}{c}
\alpha_{1}, \cdots, \alpha_{p} ; \\
\beta_{1}, \cdots, \beta_{q} ;
\end{array}-x u(t)\right]
$$

and 
(8)

$$
\begin{aligned}
\sum_{n=0}^{\infty} \frac{\alpha}{\alpha+n / 2} f_{n}^{(\alpha)}(x) t^{n} & \\
& =[1+u(t)]^{\alpha+1} F_{q+1}\left[\begin{array}{r}
2 \alpha, \alpha_{1}, \cdots, \alpha_{p} ; \\
2 \alpha+1, \beta_{1}, \cdots, \beta_{q} ;
\end{array}-x u(t)\right]
\end{aligned}
$$

where

$$
u(t)=(t / 2)\left[t+\sqrt{ }\left(t^{2}+4\right)\right]
$$

Proof. First define $u(t)$ to be the formal inverse of the function $v(t)=t / \sqrt{ }(1+t)$; that is, $u(v(t))=v(u(t))=t$. Simple computation yields the explicit expression for $u(t)$ given in (9) above. Now with $\beta=\frac{1}{2}$ and $x=u(t)$, the lemma shows that if two sequences $\phi_{n}$ and $\psi_{n}$ are related by

$$
\psi_{n}=\sum_{k=0}^{n}\left(\begin{array}{c}
\alpha+n / 2 \\
n-k
\end{array}\right) \phi_{k}
$$

then

$$
\sum_{n=0}^{\infty} \psi_{n} t^{n}=\frac{[1+u(t)]^{1+\alpha}}{1+u(t) / 2} \sum_{n=0}^{\infty} \phi_{n}[u(t)]^{n}
$$

and

$$
\sum_{n=0}^{\infty} \frac{\alpha}{\alpha+n / 2} \psi_{n} t^{n}=[1+u(t)]^{\alpha} \sum_{n=0}^{\infty} \frac{\alpha}{\alpha+n / 2} \phi_{n}[u(t)]^{n} .
$$

Finally, let

$$
\phi_{n}=\frac{\left(\alpha_{1}\right)_{n} \cdots\left(\alpha_{n}\right)_{n}}{\left(\beta_{1}\right)_{n} \cdots\left(\beta_{q}\right)_{n}} \cdot \frac{(-x)^{n}}{n !}
$$

and observe that $\psi_{n}$ in (10) becomes the polynomial $f_{*}^{(\alpha)}(x)$ defined by (6); in addition, (11) and (12) become (7) and (8) respectively. This completes the proof of the theorem.

The interesting special cases follow, and $u(t)$ is, of course, always understood to be the function in (9). Notation for the ultraspherical (Gegenbauer) polynomials varies throughout the literature [8], and we write them as $P_{s}^{(\alpha, \alpha)}(x)$ which is the form used most of ten by Brafman. Inasmuch as

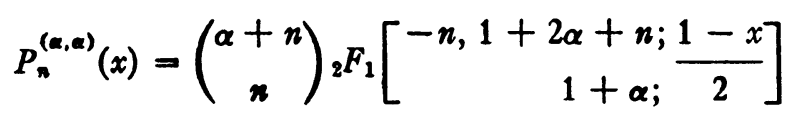


and since any ${ }_{1} F_{0}$ is a binomial expansion, we have the pair

$$
\sum_{n=0}^{\infty} P_{n}^{(\alpha-n / 2, \alpha-n / 2)}(x) t^{n}=\frac{[1+u(t)]^{1+\alpha}}{1+u(t) / 2}\left[1+\frac{1-x}{2} u(t)\right]^{-1-2 \alpha}
$$

and

$$
\sum_{n=0}^{\infty} \frac{\alpha}{\alpha+n / 2} P_{n}^{(\alpha-n / 2, \alpha-n / 2)}(x) t^{n}=[1+u(t)]^{\alpha}\left[1+\frac{1-x}{2} u(t)\right]^{-2 \alpha} .
$$

The Jacobi polynomials

$$
P_{n}^{(\alpha, \beta)}(x)=\left(\begin{array}{c}
\alpha+n \\
n
\end{array}\right){ }_{2} F_{1}\left[\begin{array}{r}
-n, 1+\alpha+\beta+n ; \frac{1-x}{2} \\
1+\alpha ;
\end{array}\right]
$$

are a direct generalization of the ultraspherical polynomials. Correspondingly, we have

$$
\sum_{n=0}^{\infty} P_{n}^{(\alpha-n / 2, \beta-n / 2)}(x) t^{n}=\frac{[1+u(t)]^{1+\alpha}}{1+u(t) / 2}\left[1+\frac{1-x}{2} u(t)\right]^{-1-\alpha-\beta}
$$

along with the hypergeometric form

$$
\begin{aligned}
\sum_{n=0}^{\infty} \frac{\alpha}{\alpha+n / 2} P_{n}^{(\alpha-n / 2, \beta-n / 2)}(x) t^{n} & \\
= & {[1+u(t)]_{2}^{\alpha} F_{1}\left[\begin{array}{r}
2 \alpha, \alpha+\beta+1 ; \\
2 \alpha+1 ;
\end{array}-\frac{1-x}{2} u(t)\right] . }
\end{aligned}
$$

Finally, recall the Laguerre polynomials

$$
L_{n}^{(\alpha)}(x)=\left(\begin{array}{c}
\alpha+n \\
n
\end{array}\right){ }_{1} F_{1}\left[\begin{array}{c}
-n ; \\
1+\alpha ;
\end{array}\right]
$$

and note that $a_{0} F_{0}$ is an exponential function. So we have

$$
\sum_{n=0}^{\infty} L_{n}^{(\alpha-n / 2)}(x) t^{n}=\frac{[1+u(t)]^{1+\alpha}}{1+u(t) / 2} \exp [-x u(t)]
$$

and the confluent hypergeometric form

$$
\sum_{n=0}^{\infty} \frac{\alpha}{\alpha+n / 2} L_{n}^{(\alpha-n / 2)}(x) t^{n}=[1+u(t)]_{1}^{\alpha} F_{1}\left[\begin{array}{c}
2 \alpha ; \\
2 \alpha+1 ;
\end{array}\right.
$$

4. Remarks. While we actually used only a special case of the lemma in $\$ 2$, the full generality of the result is of considerable interest 
in the context of a recent paper of Gould's [5]. In that paper he introduced the inverse relations

$$
\begin{aligned}
\left(\begin{array}{c}
a+b n \\
n
\end{array}\right) f(n) & =\sum_{k=0}^{n}(-1)^{k} \frac{a+b k-k}{a+b n-k}\left(\begin{array}{c}
a+b n-k \\
n-k
\end{array}\right) F(k) \\
F(n) & =\sum_{k=0}^{n}(-1)^{k}\left(\begin{array}{l}
n \\
k
\end{array}\right)\left(\begin{array}{c}
a+b k \\
n
\end{array}\right) f(k)
\end{aligned}
$$

and exhibited what he called the Vandermonde convolution transform of a certain binomial series:

$$
\sum_{n=0}^{\infty}\left(\begin{array}{c}
a+b n \\
n
\end{array}\right) f(n)\left(\frac{x-1}{x^{b}}\right)^{n}=x^{a} \sum_{n=0}^{\infty}(-1)^{n} F(n)\left(\frac{x-1}{x}\right)^{n} .
$$

It is easy to show that (3) and (2) are equivalent, respectively, to Gould's (15) and the following companion transformation which appears to be new:

$$
\begin{aligned}
& \sum_{n=0}^{\infty}\left(\begin{array}{c}
a+b n \\
n+1
\end{array}\right) f(n)\left(\frac{x-1}{x^{b}}\right)^{n} \\
& \quad=\frac{x^{a}}{x-b(x-1)} \sum_{n=0}^{\infty}(-1)^{n}(a+b n-n) F(n)\left(\frac{x-1}{x}\right)^{n} .
\end{aligned}
$$

To obtain these transformations from the lemma, set

$$
\phi_{n}=(\alpha+\beta n) F(n)
$$

and define $f(n)$ in terms of the corresponding $\psi_{n}$ by

$$
\psi_{n}=(-1)^{n}(\alpha+\beta n)\left(\begin{array}{c}
-\alpha-\beta n+n \\
n
\end{array}\right) f(n) .
$$

Then rewrite (1), (2), and (3) in terms of $f(n)$ and $F(n)$. If in the resulting equations we replace $\alpha$ by $-a, \beta$ by $1-b$, and $x$ by $(1-x) / x$, we arrive at (13), (16), and (15) respectively.

\section{REFERENCES}

1. F. Brafman, An ultraspherical generating function, Pacific J. Math. 7 (1957), 1319-1323.

2. - Generating functions of Jacobi and related polynomials, Proc. Amer. Math. Soc. 2 (1951), 942-949. 442.

3. - Series of products of Gegenbauer polynomials, Math. Z. 62 (1955), 438-

4. - Unusual generating functions for ultraspherical polynomials, Michigan Math. J. 1 (1952), 131-138. 
5. H. W. Gould, $A$ series transformation for finding convolution identities, Duke Math. J. 28 (1961), 193-202.

6. - Some generalieations of Vandermonde's convolution, Amer. Math. Monthly 63 (1956), 84-91.

7. G. P6lya and G. Szegס, Aufgaben und Lekrsutze aus der Analysis. I, SpringerVerlag, Berlin, 1964.

8. G. Szegð, Orthogonal polynomials, rev. ed., Amer. Math. Soc. Colloq. Publ. Vol. 23, Amer. Math. Soc., Providence, R. I., 1959.

Dearborn Campus, University of Michigan 